\title{
Inhibition of epithelial-mesenchymal transition in gastric cancer cells by miR-711-mediated downregulation of CD44 expression
}

\author{
WEI-SHENG XIAO $^{1 *}$, DE-FENG LI ${ }^{1-3 *}$, YA-PING TANG ${ }^{4}$, YAN-ZHU CHEN $^{5}$, WEN-BING DENG $^{1}$, \\ JUAN CHEN $^{1}$, WEI-WEI ZHOU ${ }^{1}$ and AI-JUN LIAO ${ }^{1}$ \\ ${ }^{1}$ Department of Gastroenterology, The First Affiliated Hospital of the University of South China, Hengyang, Hunan 421001; \\ ${ }^{2}$ Department of Gastroenterology, The 2nd Clinical Medical College (Shenzhen People's Hospital) of Jinan University, \\ Shenzhen, Guangdong 518020; ${ }^{3}$ Integrated Chinese and Western Medicine Postdoctoral Research Station, \\ Jinan University, Guangzhou, Guangdong 510632; ${ }^{4}$ Department of Oncology, Xiangtan Central Hospital, \\ Xiangtan, Hunan 411101; ${ }^{5}$ Department of Emergency, Shaoyang Central Hospital,
}

Shaoyang, Hunan 422000, P.R. China

Received March 1, 2018; Accepted August 17, 2018

DOI: $10.3892 /$ or.2018.6681

\begin{abstract}
Gastric cancer is a common malignancy worldwide. The prognosis of early stage gastric cancer patients has significantly improved in recent years. However, in progressive stage gastric cancer patients, the prognosis remains relatively poor due to tumor metastases. In our previous study, we showed that the expression of miR-711 in gastric cancer tissues is low, and restoration of miR-711 inhibited the invasion and migration and the occurrence of epithelial-mesenchymal transition (EMT) in gastric cancer cells. Yet, the mechanisms involved in these processes remain unknown. In the present study, we demonstrated that miR-711-mediated downregulation of CD44 expression inhibited EMT of gastric cancer cells in vitro and in vivo by downregulating vimentin protein expression and upregulating E-cadherin protein expression through transfection, qRT-PCR and western blotting. Therefore, miR-711 may provide a promising target for EMT-related therapy for gastric cancer.
\end{abstract}

\section{Introduction}

Gastric cancer is a common malignancy of the digestive tract that is highly prevalent in Asia, particularly in China and Japan. Indeed, the incidence of gastric cancer is ranked

Correspondence to: Dr Ai-Jun Liao, Department of Gastroenterology, The First Affiliated Hospital of the University of South China, 69 Chuan Shan Road, Hengyang, Hunan 421001, P.R. China E-mail: aijun.liao@163.com

\section{*Contributed equally}

Key words: CD44, E-cadherin, EMT, gastric cancer, miR-711, vimentin 4th and 2nd in malignant cancer rankings in the world and in China, respectively, and worldwide, its mortality rate remains very high (1). Although the advancement in endoscopy has significantly improved the diagnosis and prognosis of early stage gastric cancer patients, at the time of definitive diagnosis, most patients already present with lymph node and distant metastases (2). Since tumor invasion and metastasis are critical factors that affect patient prognosis, understanding the mechanisms of tumor invasion and metastasis are of utmost importance.

Previous studies have shown that a key process, known as epithelial-mesenchymal transition (EMT), occurs during tumor invasion and metastasis. EMT refers to the loss of cell polarity, intercellular adhesion and junctions, increased migratory and invasive capacities in tumor epithelial cells (3). EMT is a complex process that is characterized by increased fibrous morphology and invasiveness of cells, reduced cell apoptosis and an increase in extracellular matrix content (4). In previous studies, EMT-induced primary tumor metastases have been reported to cause poor prognosis in various cancer (5-7). Therefore, EMT is an important mechanism underlying tumor invasion and metastasis, that in recent years has become an area of intensive research.

MicroRNAs (miRNAs) are non-coding RNAs that are approximately 20-25 nucleotides in length. miRNAs are involved in several biological processes, including development, cell proliferation, cell differentiation and cell apoptosis. In addition, miRNAs are associated with the development and progression of various cancers (8). The biological effects of miRNAs are mediated through the binding of miRNAs to the 3' UTR of target mRNAs via the seed regions (the 2nd to 7th base at the $5^{\prime}$ end) and through Argonaute (Ago) protein-dependent degradation of mRNAs or blocking of mRNA translation (9). Zhang et al demonstrated that miR-181a is highly expressed in gastric cancer, and was found to promote gastric cancer cell proliferation, invasion and migration by binding to the 3' UTR of the anti-oncogene KLF6, thereby inhibiting KLF6 
expression (10). Moreover, Chen et al showed that miR-379-5p is expressed at a low level in liver cancer tissues and cells, and inhibited liver cancer cell migration, invasion and metastasis both in vitro and in vivo. Additional studies have demonstrated that miR-379-5p binds to the 3' UTR of focal adhesion kinase (FAK) to inhibit FAK expression (11). These findings have demonstrated that miRNAs play a critical role in tumor development, progression and metastasis. The recent development of miRNA microarray analysis of gastric cancer has facilitated the identification of gastric cancer development-, progression- and prognosis-related miRNAs, and includes members of the miR-200 family, miR-27, miR-373, miR-148, miR-129 and miR-711 (12-17). However, knowledge concerning these miRNAs only represent the tip of the iceberg, as the function of many miRNAs is still unknown.

In our previous study, we used miRNA microarray and identified miR-711 as an miRNA with low expression in gastric cancer. In addition, we confirmed that the expression of miR-711 in gastric cancer tissues was also low. Further investigation demonstrated that miR-711 inhibited gastric cancer cell invasion and migration, however the mechanism was unclear (17). In the present study, we demonstrated that miR-711 regulated EMT of gastric cancer cells by downregulating CD44 expression both in vivo and in vitro.

\section{Materials and methods}

Cell culture. Human gastric cancer cell lines MGC-803 and SGC-7901 provided by the Cancer Research Institute of the University of South China (Hengyang, China) were cultured in RPMI-1640 (Gibco; Thermo Fisher Scientific, Scoresby, VIC, Australia) culture medium, containing $10 \%$ fetal calf serum (FCS) in a $5 \% \mathrm{CO}_{2}$ constant-temperature incubator (Thermo Fisher Scientific, Inc., Waltham, MA, USA).

Transfection. SGC-7901 and MGC-803 human gastric cancer cells were seeded and cultured in 6 -well plates for $\sim 24 \mathrm{~h}$. Once the cells reached 40-60\% confluency, they were transfected with miR-711 mimics (Shanghai GenePharma, Co., Ltd., Shanghai, China), miR-711 inhibitor (Shanghai GenePharma, Co., Ltd.), or CD44 inhibitor (Shanghai GenePharma, Co., Ltd.) using Lipofectamine 2000 (Invitrogen; Thermo Fisher Scientific, Inc.) according to the manufacturer's instructions. Empty plasmid-transfected and non-transfected (blank) cells were used as controls. The 6 -well plates were placed into the incubator, and after $6 \mathrm{~h}$, the medium in each well was replaced with fresh $10 \%$ FCS-containing RPMI-1640 medium. The transfection efficiency was calculated at $48 \mathrm{~h}$ post-transfection, and cells with $>60 \%$ transfection efficiency were used for subsequent experiments. The transfection efficiency $=$ number of cells containing green fluorescence/a randomly observed visual field consisting of 100 cells using an optical microscope (Fig. 1).

Transwell invasion assay. After coating of the Transwell chamber with Matrigel, $500 \mu 1$ of $10 \%$ FCS-containing RPMI-1640 medium was added into the lower chamber, and $100 \mu \mathrm{l}$ of serum-free RPMI-1640 medium was added to the upper chamber. At $48 \mathrm{~h}$ post-transfection, the cells were digested with trypsin, prepared into a single-cell suspension, seeded at a density of $1 \times 10^{5}$ cells/100 $\mu \mathrm{l}$ into the upper chamber and cultured for $24 \mathrm{~h}$. Cells were fixed with $4 \%$ formaldehyde, stained with crystal violet and observed under a light microscope (Olympus Corp., Tokyo, Japan) at a magnification of x100. Ten fields were randomly chosen and the number of transmigrated cells in each field was calculated by summing the Transwell cells. The assay was performed in triplicate and the mean \pm SD of the number of transmigrated cells was calculated.

Scratch wound healing assay. At $48 \mathrm{~h}$ post-transfection, cells were digested, prepared into single-cell suspensions and seeded into 6-well plates. Once the cells reached $80 \%$ confluency, a horizontal line was scratched across each well using a pipette, and the wells were washed twice in phosphate-buffered saline (PBS). Cells were photographed at 0 and $48 \mathrm{~h}$ post-scratch using a light microscope (Olympus Corp.) to evaluate healing at the scratch site.

Dual-Luciferase reporter assay. Wild-type and mutated CD44 3' UTR vectors (Shanghai GeneChem, Co., Ltd., Shanghai China) were transfected into gastric cancer cells SGC-7901 that were transfected with miR-711 mimics or miR-711 NC (empty-transfected and used as a control) using the Dual-Glo ${ }^{\mathrm{TM}}$ Luciferase Assay System kit protocol (Promega, Madison, WI, USA). Firefly luminescence (M1) and Renilla luminescence (M2) were measured using a fluorescence detector of light signal by fluorescence microscope (Olympus Corp.).

Hematoxylin and eosin staining $(H \& E)$. Xenograft tumors were harvested and paraffin-embedded. Sections were cut and stained with hematoxylin and eosin (H\&E). Cell nuclei were stained blue-purple by hematoxylin and the cytoplasm was stained pink with eosin. Tumor cell morphology and tumor cell number were determined at a magnification of x100 using a light microscope (Olympus Corp.).

Western blot analysis. Proteins were extracted from SGC-7901 and MGC-803 gastric cancer cells and mouse xenografts using methanesulfonyl fluoride (PMSF) (Beyotime Institute of Biotechnology, Haimen, China), and the protein concentration was determined using the bicinchoninic acid (BCA) assay (Beyotime Institute of Biotechnology). Target protein bands were cut from the $5 \%$ polyacrylamide gels and immersed in transfer buffer. Polyvinylidene fluoride (PVDF) membranes were soaked in methanol for 3-5 min and transferred to transfer buffer. Proteins $(40 \mu \mathrm{g} / \mu \mathrm{l})$ were transferred to PVDF membranes and membranes were incubated in blocking buffer for $2 \mathrm{~h}$ at room temperature on a rocker at low speed. Membranes were initially incubated for $1 \mathrm{~h}$ at room temperature with anti-rabbit CD44 (bs-0521R), anti-rabbit E-cadherin (3195P) and anti-rabbit vimentin antibodies (5741P) at 1:500 dilution (Cell Signaling Technology, Danvers, MA, USA) on a shaker, and then placed overnight at $4^{\circ} \mathrm{C}$. Next, PVDF membranes were washed and then incubated with goat anti-rabbit IgG secondary antibody (11040914) at 1:1,000 dilution (Cell Signaling Technology) for 1-2 $\mathrm{h}$ at room temperature on a shaker. Proteins were visualized using a gel imaging processing and analysis system (Uvitec Ltd., Cambridge, UK). 


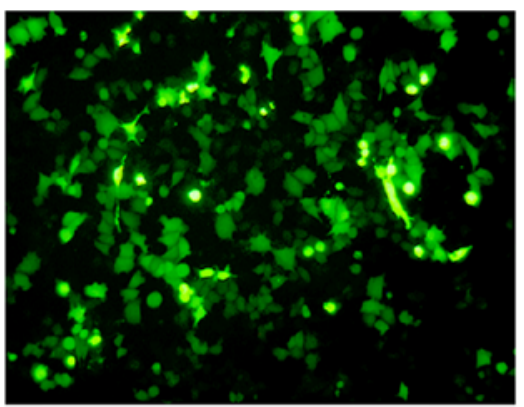

LV-miR-711 mimics

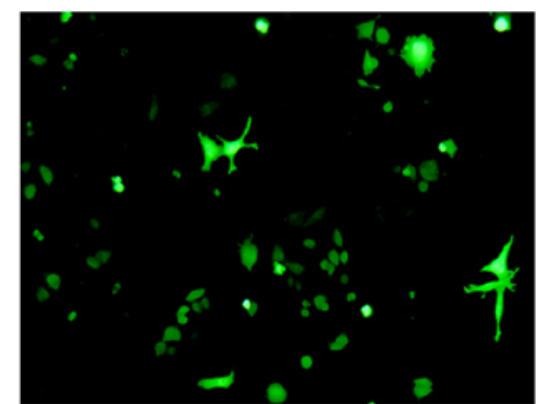

LV-NC

Figure 1. Efficiency of the miR-711 mimic transfection. Green fluorescent cells demonstrated that the transfection was successful. The transfection efficiency $=$ number of cells containing green fluorescence $/ 1$ randomly observed visual field with 100 cells under an optical microscope.

Real-time quantitative polymerase chain reaction (real-time $P C R$ ). RNA was extracted according to the RNA kit instructions (Takara Bio, Inc., Otsu, Japan) and synthesized into cDNA using a Reverse Transcription kit (Takara Bio). Next, cDNA was amplified using a real-time PCR kit (Takara Bio) in a reaction mixture containing $10 \mu \mathrm{l}$ SYBR-Green qPCR Mix, $2 \mu 1$ miR-711 primers (Applied Biosystems; Life Technologies,

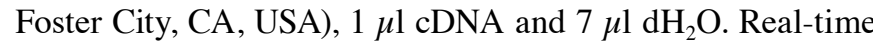
PCR was performed at $95^{\circ} \mathrm{C}$ for $3 \mathrm{~min}$, followed by $95^{\circ} \mathrm{C}$ for $10 \mathrm{sec}$, and $58^{\circ} \mathrm{C}$ for $30 \mathrm{sec}$ for a total of 40 cycles.

Tumor xenografts in nude mice. The study was approved by The First Affiliated Hospital of the University of South China Institutional Ethics Committee (no. 201708). The 10 male Balb/c nude mice (4 weeks old) provided by Beijing Vital River Laboratory Animal Technology Co., Ltd. (Beijing, China (weight, 15.5 $\pm 0.25 \mathrm{~g}$ ), were bred and housed in the same temperature and humidity-controlled room on a 12-h light/dark cycle in laminar air flow room (LAFR). The mice were provided with autoclaved tap water and autoclaved standard laboratory chow ad libitum. The gastric cancer SGC-7901 cells were cultured and then the lentivirus carrying miR-711 mimics or miR-711 NC were transfected into gastric cancer SGC-7901 cells. SGC-7901 cells with stable miR-711 mimics, or miR-711 $\mathrm{NC}$ expression were cultured for subsequent use. Harvested SGC-7901 cells $\left(1 \times 10^{7}\right)$ were subcutaneously implanted into the bilateral axilla of the mice. After 1 week, tumor volumes were measured every 7 days and the corresponding volumes were calculated by multiplying the length by the width. The mice were sacrificed after 4 weeks by decapitation as dictated by the ethical guidelines, and tumor tissues were used for further research.

Measurement of xenograft tumor volume. The length (a) and width (b) of the tumors were measured weekly, and tumor volume $(\mathrm{V})\left(\mathrm{V}=\mathrm{ab}^{2} / 2\right)$ was calculated to create a growth curve of the xenograft tumors in the nude mice. Four weeks after tumor inoculation, the mice were euthanized, solid tumors were collected, and stored at $-80^{\circ} \mathrm{C}$ for subsequent use.

Cell morphology and cell number were determined by H\&E staining. The expression of miR-711 was measured by RT-qPCR and CD44, E-cadherin and vimentin protein expression were examined by western blot analysis and immunohistochemistry.
Statistical analysis. All statistical analyses were performed using SPSS 18.0 statistical software (SPSS, Inc., Chicago, IL, USA). In this study, means and standard deviations were used for continuous variables. One-way ANOVA followed by Bonferroni was performed to test multiple variables between experimental groups, normal control groups and blank groups in Figs. 2-4. Student's t-test was performed in Figs. 5, and 7D and F. Independent samples non-parametric test was performed in xenograft tumor volume for non-normal variables. $\mathrm{P}<0.05$ was considered to indicate a statistically significant result.

\section{Results}

miR-711 inhibits gastric cancer cell invasion. The Transwell invasion assay showed that the number of transmigrated MGC-803 and SGC-7901 cells was significantly lower in the miR-711 mimics group compared to that noted in the miR-711 inhibitor $(\mathrm{P}=0.005$ and $\mathrm{P}=0.006$, respectively) and $\mathrm{NC}$ groups $(\mathrm{P}=0.02$ and $\mathrm{P}=0.03$, respectively), demonstrating that exogenous overexpression of miR-711 in SGC-7901 or MGC-803 gastric cancer cells diminished the invasiveness of these cells. Moreover, inhibition of miR-711 expression in MGC-803 or SGC-7901 cells significantly increased the number of transmigrated cells compared to the $\mathrm{NC}$, indicating that miR-711 inhibition enhanced the invasiveness of these cells $(\mathrm{P}=0.01$ and $\mathrm{P}=0.02$, respectively). There was no difference between the $\mathrm{NC}$ and blank group $(\mathrm{P}=0.96$ and $\mathrm{P}=0.63)$ (Fig. 2).

miR-711 inhibits gastric cancer cell migration. The scratch wound healing assay revealed that cancer cell migration distance and 'scratch' healing abilities were significantly reduced in the miR-711 mimics group (7.08 \pm 0.73$)$ when compared to the miR-711 NC (32.52 \pm 1.73$)$ and blank groups (32.15 \pm 1.55$)$. These findings indicated that exogenous overexpression of miR-711 significantly inhibited the migratory ability of MGC-803 or SGC-7901 cells $(\mathrm{P}=0.004, \mathrm{P}=0.001, \mathrm{P}=0.003$ and $\mathrm{P}=0.001$, respectively). In addition, inhibition of miR-711 expression enhanced the migration of MGC-803 or SGC-7901 cells, and the 'scratch' healing abilities were significantly higher in the miR-711 inhibitor group $(49.03 \pm 1.59)$ compared to that noted in the NC group $(32.52 \pm 1.73)(\mathrm{P}=0.02$ and $\mathrm{P}=0.007)$. The $\mathrm{NC}$ group was not significantly different comparing with the blank group ( $\mathrm{P}=0.28$ and $\mathrm{P}=0.87$ ) (Fig. 3). 


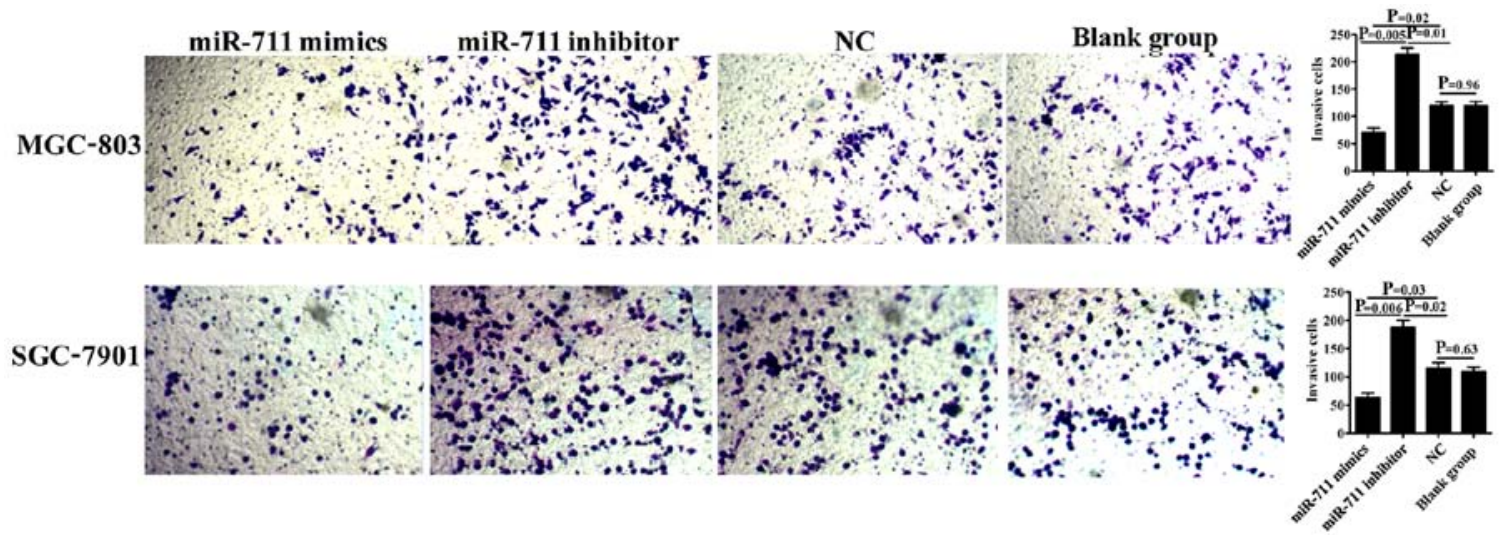

Figure 2. miR-711 inhibits gastric cancer cell invasion. (Upper panel) Exogenous overexpression of miR-711 in MGC-803 gastric cancer cells diminished the invasiveness of these cells compared to the $\mathrm{NC}$ and miR-711 inhibitor groups $(\mathrm{P}=0.02$ and $\mathrm{P}=0.005)$. Moreover, inhibition of miR-711 expression in MGC803 cells significantly increased the number of transmigrated cells compared to the $\mathrm{NC}$ group $(\mathrm{P}=0.01)$. There was no difference between the $\mathrm{NC}$ and blank group $(\mathrm{P}=0.96$. (Lower panel) At the same time, in SGC-7901 cells, exogenous overexpression of miR-711 inhibited the invasiveness of these cells compared to the $\mathrm{NC}$ and miR-711 inhibitor group $(\mathrm{P}=0.03$ and $\mathrm{P}=0.006)$. Moreover, inhibition of miR-711 expression significantly promoted the number of transmigrated cells compared to the $\mathrm{NC}$ group $(\mathrm{P}=0.02)$. There was no significant difference between the $\mathrm{NC}$ and blank group $(\mathrm{P}=0.63)$.

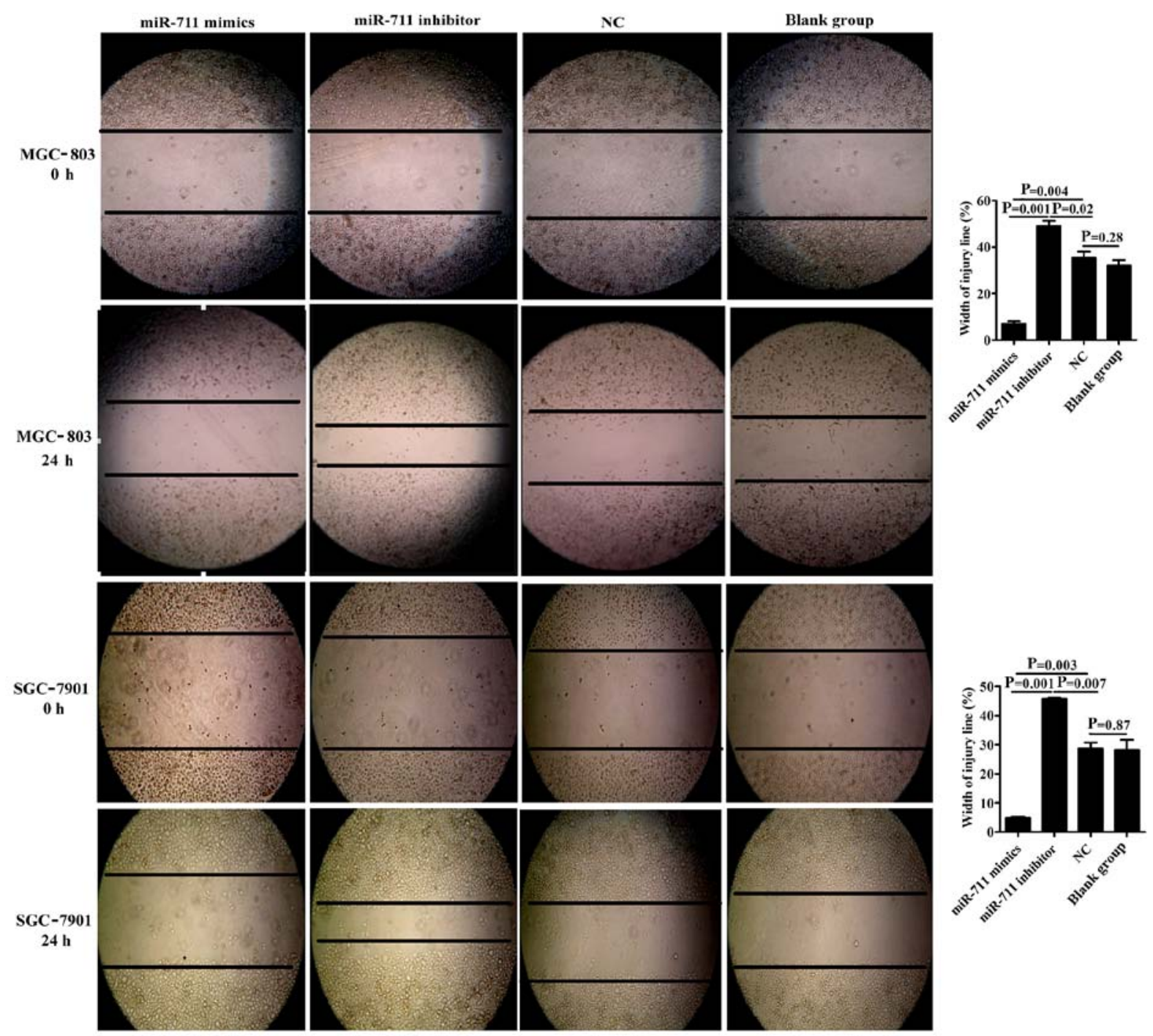

Figure 3. miR-711 inhibits gastric cancer cell migration. (Upper panels) Significantly reduced migration ability was noted in the miR-711 mimics group when compared to the $\mathrm{NC}$ and miR-711 inhibitor groups in the MGC-803 cells $(\mathrm{P}=0.004$ and $\mathrm{P}=0.001)$. In addition, inhibition of miR-711 expression enhanced the migration of MGC-803 cells compared to $\mathrm{NC}$ group $(\mathrm{P}=0.02)$. There was no difference between the $\mathrm{NC}$ and blank group $(\mathrm{P}=0.28)$. (Lower panels) Meanwhile, the results also showed that overexpression of miR-711 inhibited SGC-7901 cell migration compared with the NC and miR-711 inhibitor groups $(\mathrm{P}=0.003$ and $\mathrm{P}=0.001)$. In addition, inhibition of miR-711 in SGC-7901 cells promoted cell migration compared with the NC group ( $\mathrm{P}=0.007)$. There was no significant difference between the $\mathrm{NC}$ and blank group $(\mathrm{P}=0.87)$. 
A

MGC-803 cells

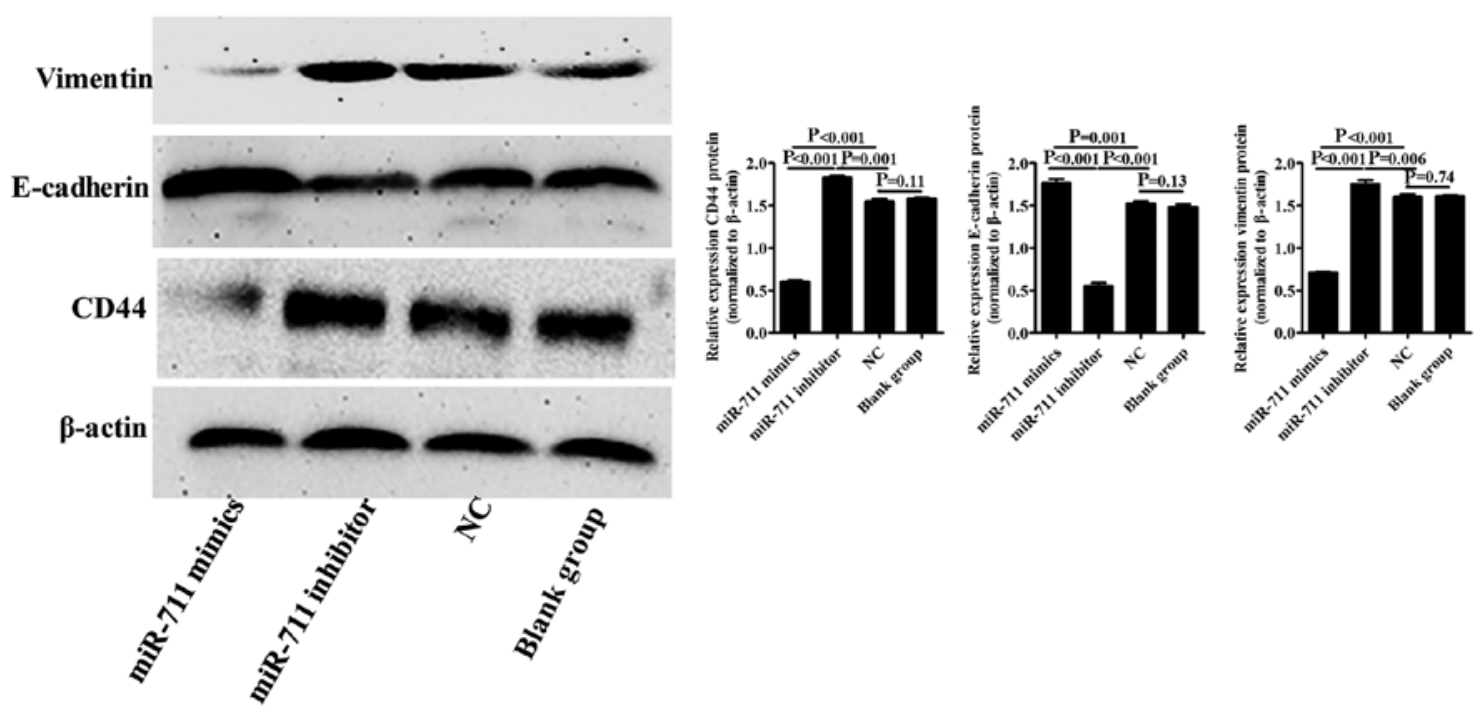

B

SGC-7901 cells
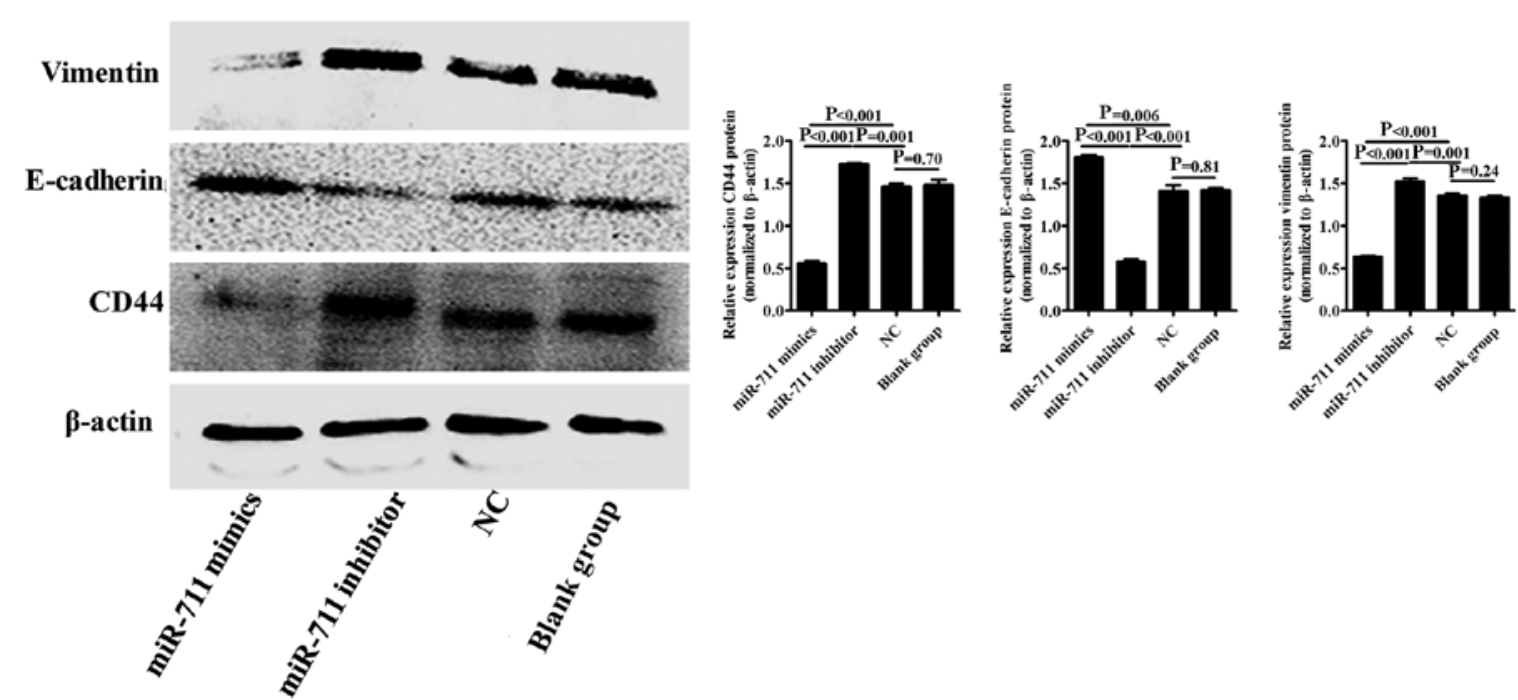

Figure 4. miR-711 regulates CD44, E-cadherin and vimentin expression. (A) In MGC-803 cells, exogenous miR-711 downregulated the CD44 and vimentin protein expression $(\mathrm{P}<0.001)$ and upregulated E-cadherin protein $(\mathrm{P}=0.001$ and $\mathrm{P}<0.001)$, compared with the NC and miR-711 inhibitor groups, while inhibition of miR-711 upregulated the expression of $\mathrm{CD} 44$ and vimentin protein expression $(\mathrm{P}=0.001$ and $\mathrm{P}=0.006)$, and inversely, downregulated E-cadherin protein $(\mathrm{P}<0.001)$. (B) In SGC-7901 cells, overexpression of miR-711 decreased CD44 and vimentin protein expression $(\mathrm{P}<0.001$ and $\mathrm{P}<0.001)$ and upregulated E-cadherin protein $(\mathrm{P}<0.001)$. However, inhibition of miR-711 upregulated the expression of $\mathrm{CD} 44$ and vimentin protein $(\mathrm{P}<0.001$ and $\mathrm{P}<0.001)$ and downregulated E-cadherin protein $(\mathrm{P}<0.001)$.

miR-711 regulates $C D 44, E$-cadherin and vimentin expression. The expression of CD44, E-cadherin and vimentin in MGC-803 or SGC-7901 cells with exogenous miR-711 overexpression or inhibition was evaluated using western blot analysis. Compared with the miR-711 NC and blank groups, CD44 and vimentin protein expression was downregulated, whereas E-cadherin protein expression was upregulated in the MGC-803 or SGC-7901 cells with miR-711 overexpression $(\mathrm{P}<0.001$ and $\mathrm{P}<0.001)$ (Fig. $4 \mathrm{~A}$ and $\mathrm{B})$. However, CD44 and vimentin protein expression were upregulated, and E-cadherin protein expression was downregulated when miR-711 was inhibited in the MGC-803 or SGC-7901 cells $(\mathrm{P}<0.001$ and $\mathrm{P}<0.001)$ (Fig. $4 \mathrm{~A}$ and $\mathrm{B})$.
$C D 44$ regulates $E$-cadherin and vimentin expression. Since CD44 has been shown to be highly expressed in gastric cancer tissues and cells, we blocked CD44 expression in MGC-803 or SGC-7901 cells and evaluated E-cadherin and vimentin expression in these cell types using western blot analysis. We found that inhibition of CD44 expression in MGC-803 or SGC-7901 cells significantly downregulated vimentin protein expression $(\mathrm{P}<0.001$ and $\mathrm{P}<0.001)$ and upregulated $\mathrm{E}$-cadherin protein expression $(\mathrm{P}=0.003$ and $\mathrm{P}<0.001)$ when compared to the NC group (Fig. 5A and B).

miR-711 specifically binds to CD44. Based on the TargetScan prediction that miR-711 specifically binds 
A

MGC -803 cells
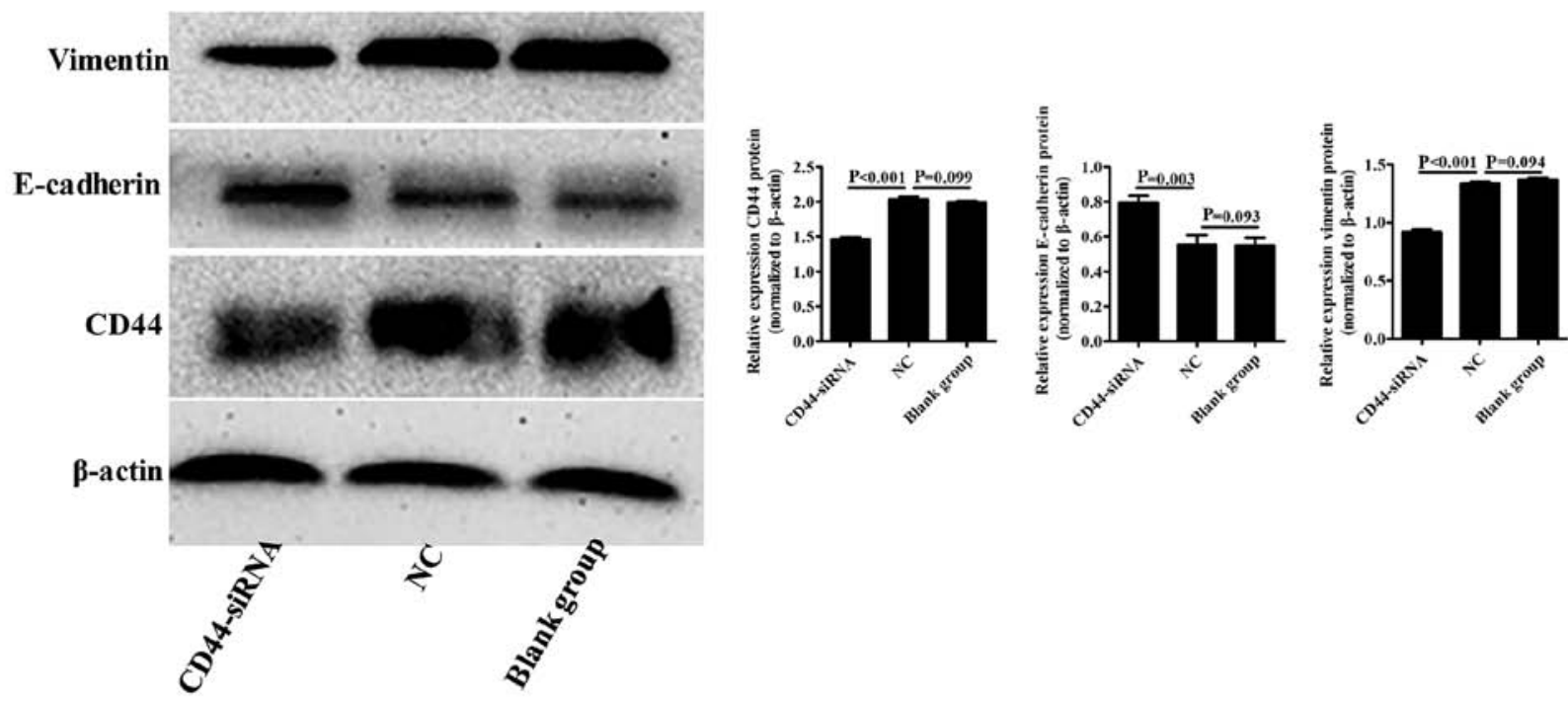

B

SGC-7901 cells
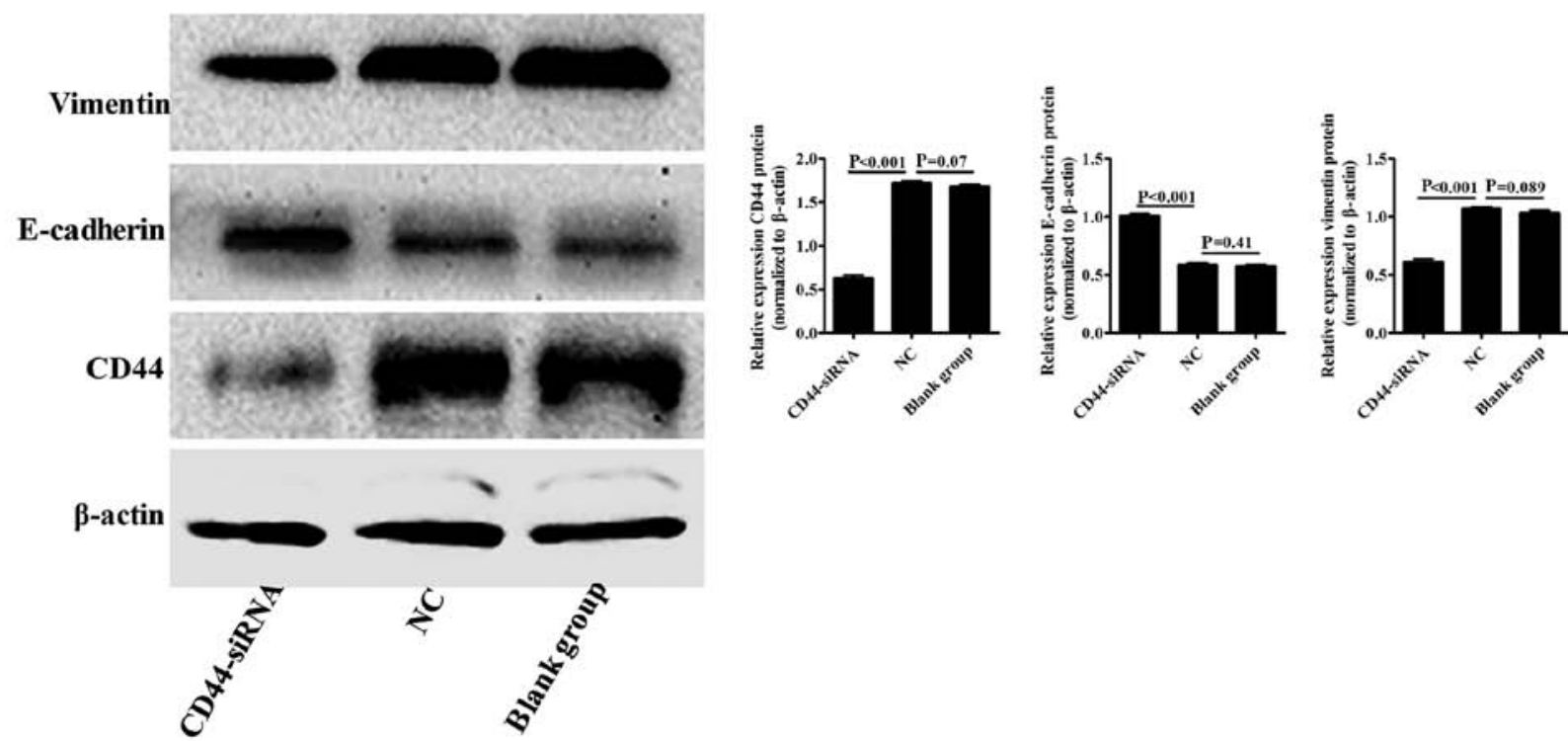

Figure 5. CD44 regulates E-cadherin and vimentin expression. (A) In MGC-803 cells, inhibition of CD44 reduced vimentin protein (P<0.001), but, increased E-cadherin protein $(\mathrm{P}=0.003)$. (B) The results showed that downregulation of CD44 in SGC-7901 cells, also downregulated vimentin protein $(\mathrm{P}<0.001)$, however, upregulated E-cadherin protein $(\mathrm{P}<0.001)$.

to CD44 3' UTR (Fig. 6A), we constructed CD44 3' UTR-wild (wild-type) and CD44 3'-UTR-mut (mutant) vectors and co-transfected SGC-7901 cells with either vectors and miR-711 mimics. Dual-Luciferase reporter assays revealed that in the CD44 3' UTR-wild group, luciferase activity was significantly reduced compared to the CD44 3' UTR-mut group $(\mathrm{P}=0.009)$ (Fig. 6B).

miR-711-mediated upregulation of CD44 inhibits EMT in gastric cancer in vivo. SGC-7901 gastric cancer cells with stable miR-711 mimcs (overexpression) or miR-711 NC were grafted into nude mice under the armpit, and nude mice were euthanized 4 weeks post-grafting. Tumor formation assay indicated that the xenograft tumor volume was significantly smaller in the miR-711 mimics group compared to that in the miR-711 NC group $(\mathrm{P}<0.001)$ at day 28 (Fig. 7A-C). Moreover,
qRT-PCR revealed that miR-711 expression was significantly higher in the xenograft tumors with miR-711 overexpression when compared to the NC group $(\mathrm{P}=0.0008)$ (Fig. 7D). Immunohistochemistry using H\&E staining showed that, at each magnification used, the number of tumor cells was significantly lower in the miR-711 mimics group when compared to the miR-711 NC group $(\mathrm{P}<0.05)$ (Fig. 7E). Furthermore, western blot analysis demonstrated that CD44 and vimentin protein expression levels were significantly downregulated, and E-cadherin protein expression was significantly upregulated in the xenograft tumors in the miR-711 mimics group compared to those in the miR-711NC group $(\mathrm{P}=0.015$, $\mathrm{P}=0.035$ and $\mathrm{P}=0.028$, respectively) (Fig. $7 \mathrm{~F}$ ). Therefore, it was discovered that miR-711 can inhibit cell invasion and migration in vitro, and inhibit cell proliferation and xenograft tumor growth in vivo. 


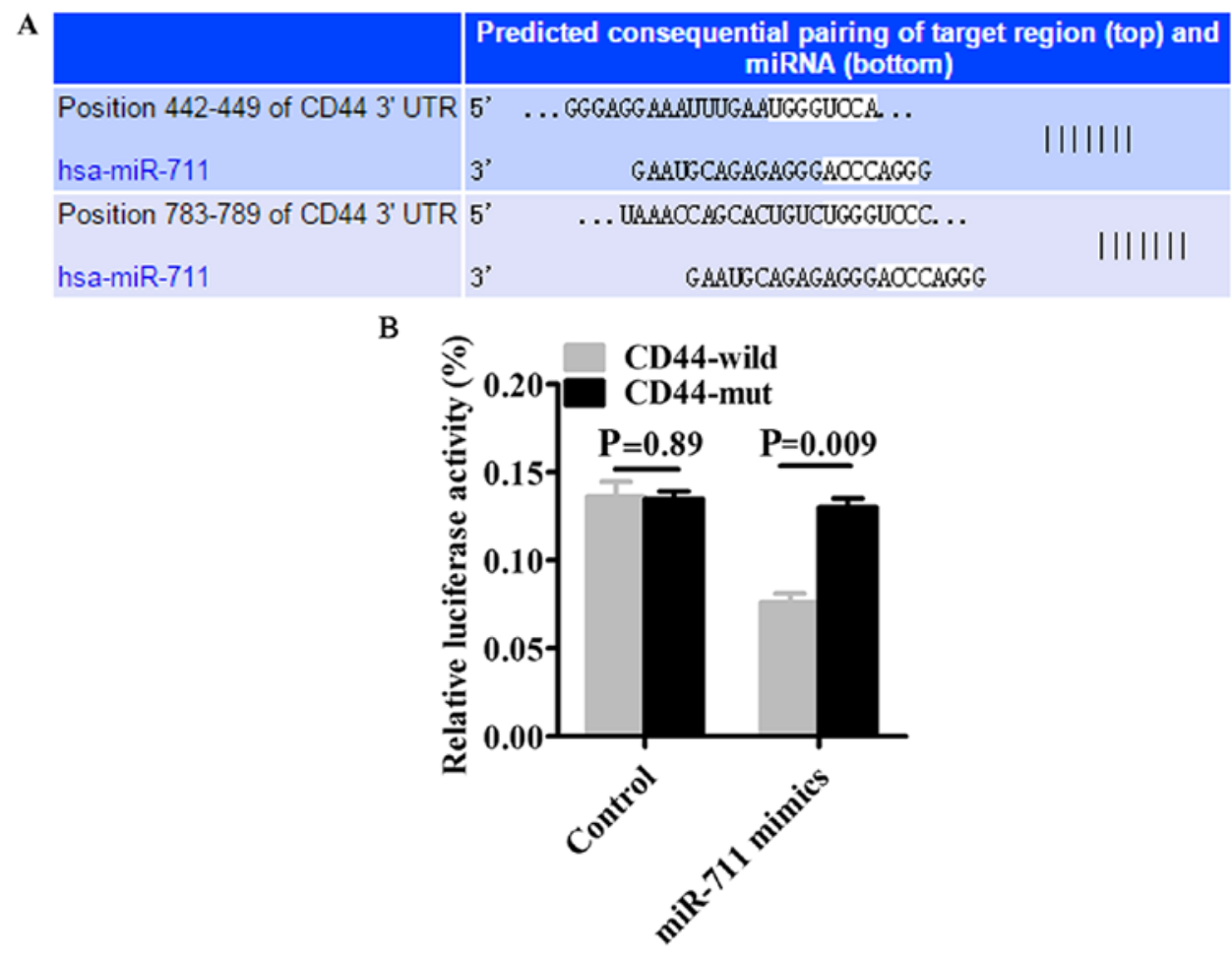

Figure 6. miR-711 specifically binds to CD44. (A) miR-711 was found to specifically bind to CD44 3' UTR by using bioinformatic analysis. (B) SGC-7901 cells were co-transfected with miR-711 mimics or control, Renilla luciferase vector pRL-SV40, and full length CD44 3' UTR luciferase reporter for $48 \mathrm{~h}$. Both firefly luciferase signals were normalized with the Renilla luciferase signals. The results revealed that in the CD44 3' UTR-wild group, luciferase activity was significantly reduced compared to the CD44 3' UTR-mut group ( $\mathrm{P}=0.009)$.

A

\section{B}
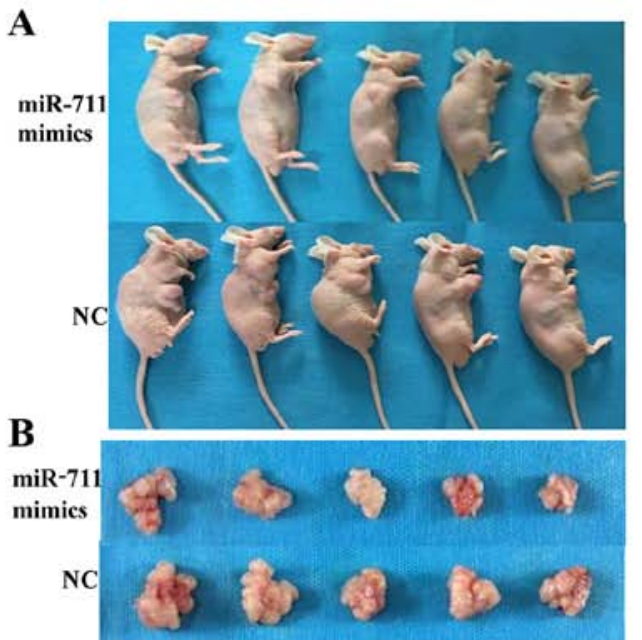

C

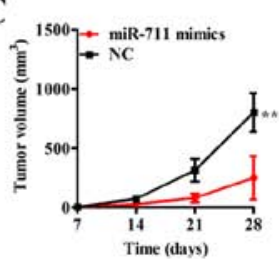

D

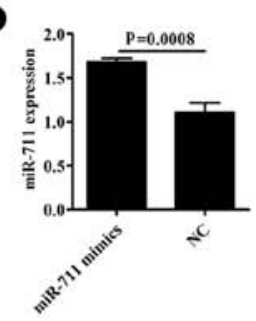

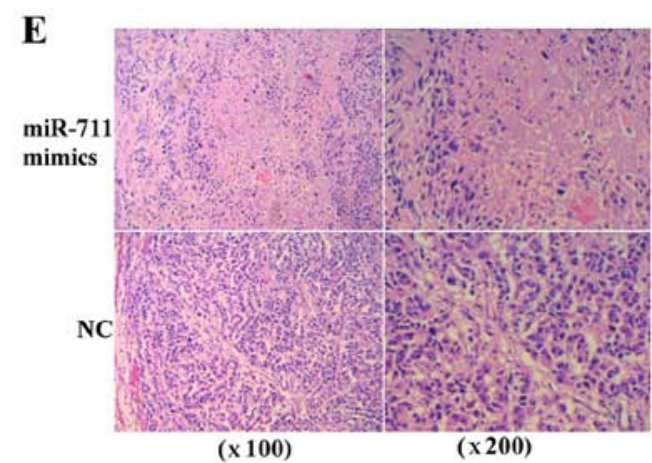

$\mathbf{F}$

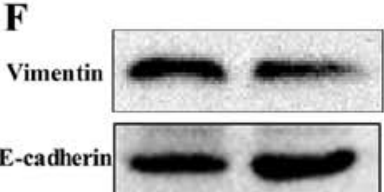

CD44
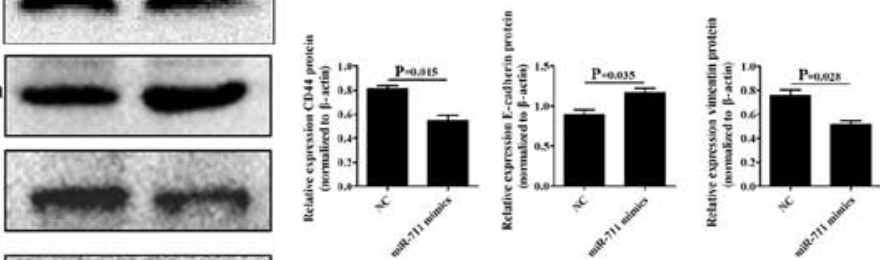

$\beta$-actin

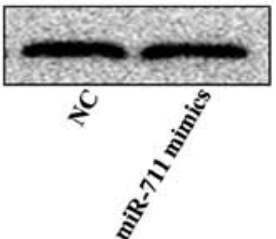

Figure 7. miR-711-mediated downregulation of CD44 inhibits EMT in gastric cancer in vivo. (A-C) Tumor formation assay indicated that the xenograft tumor volume was significantly smaller in the miR-711 mimics group compared to the NC group (P<0.001). (D) qRT-PCR revealed that miR-711 expression was significantly higher in xenograft tumors with miR-711 overexpression when compared to the NC group $(\mathrm{P}=0.0008)$. (E) H\&E staining showed that the number of tumor cells was significantly increased in the miR-711 mimics group compared to the NC group (P<0.05). (F) Western blot analysis demonstrated that CD44 and vimentin protein expression was significantly downregulated, and E-cadherin protein expression was significantly upregulated in xenograft tumors in the miR-711 mimics group compared to those in the $\mathrm{NC}$ group $(\mathrm{P}=0.015, \mathrm{P}=0.035$ and $\mathrm{P}=0.028$, respectively). 


\section{Discussion}

Over $90 \%$ of cancer-related deaths are associated with tumor epithelial-mesenchymal transition (EMT). Regulation of tumor cells by EMT-related proteins during tumor development and progression results in weakened intercellular adhesion, varying degrees of mesenchymal phenotypes, and enhanced mobility and invasiveness. This allows cells to cross the basement membrane with the help of proteases in the surrounding microenvironment, and results in local infiltration and distant metastasis. Tumor cells that arrive at the site of metastatic lesions can reverse EMT and re-transform into tumor epithelial cells, which then proliferate to form distant metastatic lesions and promote tumor progression (18-21). Several factors induce or regulate the biological processes of EMT, such as the tumor microenvironment, cytokines, signaling kinases and transcription factors. Moreover, changes in EMT-related proteins including the expression of epithelial cell adhesion proteins, cell morphology-related proteins and mesenchymal cell-related proteins, such as E-cadherin and vimentin have also been reported (22-25). In fact, several studies have demonstrated that the biological processes of EMT play a critical role in the development, progression, invasion and migration.

EMT is often accompanied by an increase in cancer stem cells (CSCs), and is therefore believed to be one of the inducing factors for CSC formation (26). Previous studies have suggested that prostate CSCs may be the source of prostate cancer and that they have an important role in the development and progression of prostate cancer. Prostate CSCs can be identified by several unique markers, including CD133, CD44, BCRP-1/ABG2 and telomerase $(27,28)$. Ishimoto et al found that CD44 is an important marker for CSCs (29). In another study, it was demonstrated that the use of an anti-CD44 monoclonal antibody eliminated acute myeloid leukemia (AML) CSCs (30). CD44 is a multifunctional cell surface adhesion receptor that plays a key role in the invasion and metastasis of multiple cancers. In vitro and in vivo studies have revealed that miR-647 may inhibit gastric cancer metastasis via inhibition of CD44 expression (31). In a study by Lee et al, it was reported that downregulation of CD44 expression in HCT116 colon cancer cells inhibited cancer cell proliferation, migration and invasion and promoted cell apoptosis (32). Moreover, it was found that exogenous asporin bound to the CD44 receptor on pancreatic cancer cells to downregulate epithelial phenotype-related proteins and upregulate mesenchymal phenotype-related proteins, which induced tumor cell EMT, and thereby promoted tumor cell invasion and migration (33).

At present, studies on the biological roles of miR-711 are limited. In a previous study, it was shown that pioglitazone inhibited collagen-I synthesis and post-infarction myocardial fibrosis by upregulating miR-711 expression in cardiomyocytes (34). miR-711-mediated upregulation of adiponectin (ApN) inhibited Toll-like receptor 4 (TLR4) signaling and thereby suppressed inflammation during myositis (35). In a recent study by $\mathrm{Hu}$ et al, a new role of miR-711 in cancer was revealed. Specifically, the authors showed that high miR-711 expression in breast cancer tissues was a risk factor for breast cancer patients, and that high miR-711 expression promoted breast cancer cell proliferation, invasion and migration in vitro, suggesting that miR-711 served as an oncogene (36). However, Waseem et al found that lower miR-711 expression in prostate cancer resulted in a higher Gleason score, greater malignancy, and higher cancer metastatic rate, indicating that miR-711 was an anti-oncogene (37). Because of the inconsistent findings, the biological functions of miR-711 remain unclear. In our previous study, we showed that the expression of miR-711 in gastric cancer tissues and cells was low, and that exogenous miR-711 expression inhibited gastric cancer cell invasion and migration by an unidentified mechanism (17). In this study, exogenous miR-711 overexpression in SGC-7901 or MGC-803 gastric cancer cells inhibited cancer cell invasion and migration. In contrast, inhibition of miR-711 expression promoted gastric cancer invasion and migration, indicating that miR-711 inhibited gastric cancer progression. Moreover, exogenous miR-711 overexpression can inhibit xenograft tumor growth in vivo and may therefore be an anti-oncogene. It was discovered that the process of EMT is dispensable for metastasis, and caused chemo-resistance in lung and pancreatic cancer $(38,39)$. In this study, EMT contributed to metastasis in gastric cancer. Since bioinformatics shows that miR-711 specifically binds to the 3' UTR of CD44, we speculated that the biological roles of miR-711 may be mediated through CD44. To test this hypothesis, we performed western blot analysis and confirmed that exogenous miR-711 overexpression inhibited CD44 and vimentin protein expression and promoted E-cadherin protein expression. In contrast, inhibition of miR-711 expression promoted CD44 and vimentin protein expression and inhibited E-cadherin protein expression. These findings indirectly demonstrated that miR-711 may inhibit EMT in gastric cancer by regulating CD44, vimentin and E-cadherin expression. In a previous study, CD44 was shown to be highly expressed in gastric cancer tissues and cells, and was involved in the regulation of gastric cancer metastasis (40). We inhibited CD44 expression in SGC-7901 and MGC-803 gastric cancer cells and demonstrated that CD44 inhibition downregulated vimentin protein expression and upregulated E-cadherin protein expression. Therefore, we believed that miR-711 may inhibit EMT in gastric cancer by downregulating CD44 expression, which in turn inhibited vimentin expression and promoted E-cadherin expression. Further investigation using dual-fluorescence assays revealed that the relative luciferase activity was significantly lower in miR-711 mimics-transfected CD44 3' UTR-wt SGC-7901 cells compared to miR-711 mimics-transfected CD44 3' UTR-mut SGC-7901 cells, thereby indicating that miR-711 specifically bound to the CD44 3' UTR. It further demonstrated that miR-711 inhibited EMT in gastric cancer via inhibition of CD44 3' UTR. To further confirm our hypothesis, we cultured and grafted SGC-7901 cells with stable miR-711 overexpression into nude mice, and showed that exogenous miR-711 overexpression significantly inhibited xenograft tumor growth in the miR-711 mimics group compared to the $\mathrm{NC}$ group, demonstrating that miR-711 inhibited xenograft tumor growth in nude mice. In addition, H\&E staining showed that the number of tumor cells was significantly reduced in the miR-711 mimics group compared to that in the NC group, indicating that miR-711 indeed inhibited tumor growth. qRT-PCR confirmed that miR-711 expression was significantly elevated in the miR-711 mimics group when compared to the NC group. Moreover, 
western blot analyses demonstrated that exogenous miR-711 overexpression inhibited EMT in gastric cancer by inhibiting CD44 and vimentin protein expression and by promoting E-cadherin protein. In summary, this study was the first to show that miR-711-mediated inhibition of CD44 expression inhibited EMT of gastric cancer cells in vitro and in vivo via downregulation of vimentin expression and upregulation of E-cadherin expression. Thus, our findings provide novel insights into the development of miR-711-based targeted therapy for EMT in gastric cancer.

\section{Acknowledgements}

We wish to thanks Professor Qi Su the for technical support.

\section{Funding}

The present study was supported by the Natural Science Foundation of Hunan Province (nos. 2017JJ3270 and 2018JJ2356), the Three Engineering Training Funds in Shenzhen (nos. SYLY201718 and SYJY201801) and the Hunan Provincial 2018 Annual Clinical Key Specialist Construction Project (no. 12).

\section{Availability of data and materials}

The datasets used during the present study are available from the corresponding author upon reasonable request.

\section{Authors' contributions}

WSX, AJL and DFL conceived and designed the study. YPT, YZC, WBD and JC completed the experiments and collected the data. WWZ, YPT, YZC, WBD and JC conducted the statistical analysis. DFL wrote the manuscript. AJL, WWZ and WSX revised the manuscript. AJL and DFL obtained funding. All authors approved the manuscript and agree to be accountable for all aspects of the research in ensuring that the accuracy or integrity of any part of the work are appropriately investigated and resolved.

\section{Ethics approval and consent to participate}

The study was approved by The First Affiliated Hospital of the University of South China Institutional Ethics Committee (no. 201708).

\section{Patient consent for publication}

Not applicable.

\section{Competing interests}

The authors declare that they have no competing interests.

\section{References}

1. Torre LA, Bray F, Siegel RL, Ferlay J, Lortet-Tieulent J and Jemal A: Global cancer statistics, 2012. CA Cancer J Clin 65: $87-108,2015$
2. Martin-Richard M, Custodio A, Garcia-Giron C, Gravalos C, Gomez C, Jimenez-Fonseca P, Manzano JL, Pericay C, Rivera F and Carrato A: Seom guidelines for the treatment of gastric cancer 2015. Clin Transl Oncol 17: 996-1004, 2015.

3. Thiery JP, Acloque H, Huang RY and Nieto MA: Epithelialmesenchymal transitions in development and disease. Cell 139: 871-890, 2009

4. Yan W, Cao QJ, Arenas RB, Bentley B and Shao R: GATA3 inhibits breast cancer metastasis through the reversal of epithelial-mesenchymal transition. J Biol Chem 285: 14042-14051, 2010.

5. Chen Y, Liu J, Wang W, Xiang L, Wang J, Liu S, Zhou H and Guo Z: High expression of hnRNPA1 promotes cell invasion by inducing EMT in gastric cancer. Oncol Rep 39: 1993-1701, 2018.

6. Huang M, Wu S, Hu Q, Wu H, Wei S, Xie H, Sun K, Li X and Fang L: Agkihpin, a novel SVAE may inhibit the migration and invasion of liver cancer cells associated with the inversion of EMT induced by Wnt/beta-catenin signaling inhibition. Biochem Biophys Res Commun 479: 283-289, 2016.

7. Wang J, Wang X, Liu F and Fu Y: microRNA-335 inhibits colorectal cancer HCT116 cells growth and epithelialmesenchymal transition (EMT) process by targeting Twist1. Pharmazie 72: 475-481, 2017.

8. Ventura A and Jacks T: MicroRNAs and cancer: Short RNAs go a long way. Cell 136: 586-591, 2009.

9. Bartel DP: MicroRNAs: Target recognition and regulatory functions. Cell 136: 215-233, 2009.

10. Zhang X, Nie Y, Du Y, Cao J, Shen B and Li Y: MicroRNA-181a promotes gastric cancer by negatively regulating tumor suppressor KLF6. Tumour Biol 33: 1589-1597, 2012.

11. Chen JS, Li HS, Huang JQ, Dong SH, Huang ZJ, Yi W, Zhan GF, Feng JT, Sun JC and Huang XH: MicroRNA-379-5p inhibits tumor invasion and metastasis by targeting FAK/AKT signaling in hepatocellular carcinoma. Cancer Lett 375: 73-83, 2016.

12. Song F, Yang D, Liu B, Guo Y, Zheng H, Li L, Wang T, Yu J, Zhao Y, Niu R, et al: Integrated microRNA network analyses identify a poor-prognosis subtype of gastric cancer characterized by the miR-200 family. Clin Cancer Res 20: 878-889, 2014.

13. Zhang Z, Liu S, Shi R and Zhao G: miR-27 promotes human gastric cancer cell metastasis by inducing epithelial-to-mesenchymal transition. Cancer Genet 204: 486-491, 2011.

14. Shi Y, Shi H, Zhang B, Yan Y, Han X, Jiang W, Qian H and Xu W: miR-373 suppresses gastric cancer metastasis by downregulating vimentin. Mol Med Rep 17: 4027-4034, 2018.

15. Chen X, Wang G, Lu X, Gao P, Song Y, Sun J, Li A, Xu Y, Xu H and Wang Z: Polymorphisms and haplotypes of the miR-148/152 family are associated with the risk and clinicopathological features of gastric cancer in a Northern Chinese population. Mutagenesis 29: 401-407, 2014.

16. Yu X, Luo L, Wu Y, Yu X, Liu Y, Yu X, Zhao X, Zhang X, Cui L, Ye G, et al: Gastric juice miR-129 as a potential biomarker for screening gastric cancer. Med Oncol 30: 365, 2013.

17. Liao A, Tan G, Chen L, Zhou W and Hu H: RASSF1A inhibits gastric cancer cell proliferation by miR-711-mediated downregulation of CDK4 expression. Oncotarget 7: 5842-5851, 2016.

18. Ma Z, Xin Z, Hu W, Jiang S, Yang Z, Yan X, Li X, Yang Y and Chen F: Forkhead box O proteins: Crucial regulators of cancer EMT. Semin Cancer Biol 50: 21-31, 2018.

19. Boulding T, McCuaig RD, Tan A, Hardy K, Wu F, Dunn J, Kalimutho M, Sutton CR, Forwood JK, Bert AG, et al: LSD1 activation promotes inducible EMT programs and modulates the tumour microenvironment in breast cancer. Sci Rep 8: 73, 2018.

20. Zhang Z, Zou Y, Liang M, Chen Y, Luo Y, Yang B, Liu F, Qin Y, He D, Wang F and Huang O: Suppressor of fused (Sufu) promotes epithelial-mesenchymal transition (EMT) in cervical squamous cell carcinoma. Oncotarget 8: 114226-114238, 2017.

21. Zhang J, Tian XJ, Zhang H, Teng Y, Li R, Bai F, Elankumaran S and Xing J: TGF-beta-induced epithelial-to-mesenchymal transition proceeds through stepwise activation of multiple feedback loops. Sci Signal 7: ra91, 2014.

22. Asakura T, Yamaguchi N, Ohkawa K and Yoshida K: Proteasome inhibitor-resistant cells cause EMT-induction via suppression of E-cadherin by miR-200 and ZEB1. Int J Oncol 46: 2251-2260, 2015.

23. Qin Y, Tang B, Hu CJ, Xiao YF, Xie R, Yong X, Wu YY, Dong $H$ and Yang SM: An hTERT/ZEB1 complex directly regulates E-cadherin to promote epithelial-to-mesenchymal transition (EMT) in colorectal cancer. Oncotarget 7: 351-361, 2016. 
24. Satelli A, Batth I, Brownlee Z, Mitra A, Zhou S, Noh H, Rojas CR, Li H, Meng QH and Li S: EMT circulating tumor cells detected by cell-surface vimentin are associated with prostate cancer progression. Oncotarget 8: 49329-49337, 2017.

25. Zhai X, Zhu H, Wang W, Zhang S, Zhang Y and Mao G: Abnormal expression of EMT-related proteins, S100A4, vimentin and E-cadherin, is correlated with clinicopathological features and prognosis in HCC. Med Oncol 31: 970, 2014.

26. Ikezono Y, Koga H, Akiba J, Abe M, Yoshida T, Wada F, Nakamura T, Iwamoto H, Masuda A, Sakaue T, et al: Pancreatic neuroendocrine tumors and EMT behavior are driven by the CSC marker DCLK1. Mol Cancer Res 15: 744-752, 2017.

27. Jayaraman A, Kumar P, Marin S, de Atauri P, Mateo F, M Thomson T, J Centelles J, F Graham S and Cascante M: Untargeted metabolomics reveals distinct metabolic reprogramming in endothelial cells co-cultured with CSC and non-CSC prostate cancer cell subpopulations. PLoS One 13: e0192175, 2018.

28. MateoF,Meca-Cortes O,Celia-TerrassaT,FernandezY,AbasoloI, Sanchez-Cid L, Bermudo R, Sagasta A, Rodriguez-Carunchio L, Pons M, et al: SPARC mediates metastatic cooperation between CSC and non-CSC prostate cancer cell subpopulations. Mol Cancer 13: 237, 2014

29. Ishimoto T, Oshima H, Oshima M, Kai K, Torii R, Masuko T, Baba $\mathrm{H}$, Saya $\mathrm{H}$ and Nagano O: $\mathrm{CD} 44^{+}$slow-cycling tumor cell expansion is triggered by cooperative actions of Wnt and prostaglandin E2 in gastric tumorigenesis. Cancer Sci 101: 673-678, 2010.

30. Gadhoum SZ, Madhoun NY, Abuelela AF and Merzaban JS: Anti-CD44 antibodies inhibit both mTORC1 and mTORC2: A new rationale supporting CD44-induced AML differentiation therapy. Leukemia 30: 2397-2401, 2016.

31. Cao W, Wei W, Zhan Z, Xie D, Xie Y and Xiao Q: Role of miR-647 in human gastric cancer suppression. Oncol Rep 37: 1401-1411, 2017.
32. Lee SY, Kim KA, Kim CH, Kim YJ, Lee JH and Kim HR: CD44-shRNA recombinant adenovirus inhibits cell proliferation, invasion, and migration, and promotes apoptosis in HCT116 colon cancer cells. Int J Oncol 50: 329-336, 2017.

33. Wang L, Wu H, Wang L, Zhang H, Lu J, Liang Z and Liu T: Asporin promotes pancreatic cancer cell invasion and migration by regulating the epithelial-to-mesenchymal transition (EMT) through both autocrine and paracrine mechanisms. Cancer Lett 398: 24-36, 2017.

34. Zhao N, Yu H, Yu H, Sun M, Zhang Y, Xu M and Gao W: MiRNA-711-SP1-collagen-I pathway is involved in the anti-fibrotic effect of pioglitazone in myocardial infarction. Sci China Life Sci 56: 431-439, 2013.

35. Boursereau R, Abou-Samra M, Lecompte S, Noel L and Brichard SM: New targets to alleviate skeletal muscle inflammation: Role of microRNAs regulated by adiponectin. Sci Rep 7: 43437, 2017.

36. Hu JY, Yi W, Zhang MY, Xu R, Zeng LS, Long XR, Zhou XM, Zheng XS, Kang Y and Wang HY: MicroRNA-711 is a prognostic factor for poor overall survival and has an oncogenic role in breast cancer. Oncol Lett 11: 2155-2163, 2016.

37. Waseem M, Ahmad MK, Srivatava VK, Rastogi N, Serajuddin M, Kumar S, Mishra DP, Sankhwar SN and Mahdi AA: Evaluation of miR-711 as novel biomarker in prostate cancer progression. Asian Pac J Cancer Prev 18: 2185-2191, 2017.

38. Zheng X, Carstens JL, Kim J, Scheible M, Kaye J, Sugimoto H, Wu CC, LeBleu VS and Kalluri R: Epithelial-to-mesenchymal transition is dispensable for metastasis but induces chemoresistance in pancreatic cancer. Nature 527: 525-530, 2015.

39. Fischer KR, Durrans A, Lee S, Sheng J, Li F, Wong ST, Choi H, El Rayes T, Ryu S, Troeger J, et al: Epithelial-to-mesenchymal transition is not required for lung metastasis but contributes to chemoresistance. Nature 527: 472-476, 2015.

40. Hu Y, Wang J, Qian J, Kong X, Tang J, Wang Y, Chen H, Hong J, Zou W, Chen Y, et al: Long noncoding RNA GAPLINC regulates CD44-dependent cell invasiveness and associates with poor prognosis of gastric cancer. Cancer Res 74: 6890-6902, 2014 